\title{
Adherencia a las intervenciones dietéticas en pacientes con diabetes mellitus tipo 2: una revisión narrativa de la literatura
}

\author{
Adherence to dietary interventions in patients with Type 2 Diabetes Mellitus: a \\ narrative review of the literature \\ Adesão às intervenções dietéticas em pacientes com Diabetes Mellitus 2: uma revisão \\ narrativa da literatura
}

Claudia N. Orozco-González* , Carolina Vieyra-Angeles², Marina Idalia Rojo-López³ ${ }^{3}$ Leticia Aguilar-Aguayo ${ }^{4}$, Saby Camacho-López ${ }^{5}$.

Recibido: 4 de julio de 2020. Aceptado para publicación: 29 de diciembre de 2020.

Publicado en línea: 18 de febrero de 2021.

https://doi.org/10.35454/rncm.v4n3.172

\section{Resumen}

Introducción: la diabetes mellitus tipo 2 (DM2) es uno de los principales problemas epidemiológicos debido a la naturaleza compleja de la enfermedad. En el plano de atención individual, es necesario llevar a cabo intervenciones nutricionales y promover hábitos de alimentación saludables, utilizando herramientas que permitan una adecuada adherencia a dichas medidas. El objetivo de esta revisión es identificar, a través del análisis de la literatura, las estrategias dietéticas para el control de la DM2 y compararlas según su eficacia para lograr la adherencia del paciente.

Métodos: se consultaron las bases de datos Pubmed, Scielo, Google Académico, Ovid y Web of Science con las palabras clave.

Resultados: la educación a largo plazo provee conocimientos que el paciente puede utilizar como forma de empoderamiento para la toma de decisiones; sin embargo, estos estudios demostraron cómo la libertad de elegir los alimentos disminuye de manera considerable la adherencia nutricional. La intervención a través de menús establecidos o un control estricto genera buenos resultados a corto plazo, pero apenas logran la consciencia que permitirá al paciente ser responsable de la enfermedad. Este tipo de intervención pa-

\section{Summary}

Background: Type 2 Diabetes Mellitus (DM2) is a major epidemiological problem due to the complex nature of the disease. At individual care level, it is essential to carry out nutritional interventions and promote healthy eating habits, using tools that allow adequate adherence to these measures. The objective of this review is to identify dietary strategies to manage DM2 and compare them according to their efficacy to achieve patient adherence through a literature analysis.

Methods: PUBMED, SciELO, Google Academic, Ovid and Web of Science databases were searched using the keywords "Diabetes type", "nutrition", "treatment", "adherence", "menu", "meal plan", "Diet", and "strict diet".

Results: Long-term education provides information that the patient can use as a form of empowerment for decisionmaking. However, the analyzed studies showed how freedom to choose food significantly reduces nutritional adherence. An intervention involving fixed menus, or a strict control, generates good results in the short term, but hardly generates enough awareness to allow the patient to be accountable for his or her condition. This type of intervention appears to do

\section{Resumo}

Introdução: O Diabetes Mellitus 2 (DM2) é um dos principais problemas epidemiológicos devido à natureza complexa da doença. No nível de atenção individual, é necessário realizar intervenções nutricionais e promover hábitos alimentares saudáveis, utilizando ferramentas que permitam a adesão adequada a essas medidas. O objetivo desta revisão é identificar, por meio da análise da literatura, as estratégias nutricionais para o controle do DM2 e compará-las quanto à eficácia para alcançar a adesão do paciente.

Métodos: foram consultadas as bases de dados PUBMED, SciELO, Google Scholar, Ovid e Web of Science com as palavras-chave "Diabetes type", "nutrition", "treatment", "adherence", "menu", "meal plan", "diet" y"strict diet".

Resultados: a educação ao longo prazo fornece conhecimentos que o paciente pode usar como referência para tomada de decisões, no entanto, esses estudos demonstraram como a liberdade de escolha dos alimentos reduz consideravelmente a adesão ao tratamento nutricional. A intervenção por meio de cardápios estabelecidos ou controle rigoroso gera bons resultados em curto prazo, mas dificilmente alcançam a consciência que 
rece hacer poco para ayudar al individuo a analizar la magnitud e importancia de modificar los hábitos dietéticos de forma saludable a largo plazo.

Conclusión: la intervención constante a través de la educación en nutrición es efectiva en la tarea de crear adherencia de los pacientes con DM2 a tratamientos nutricionales a largo plazo.

Palabras clave: intervenciones dietéticas, diabetes mellitus, adherencia, alimentos y educación en nutrición, evaluación, eficacia. little to help individuals understand the magnitude and significance of the longterm modification of dietary habits in a healthy way.

Conclusion: Constant intervention through nutrition education is effective in creating adherence to long-term nutritional approaches in patients with DM2.

Keywords: Dietary Interventions; Diabetes Mellitus; Adherence, Food and Nutrition Education; Evaluation; Effectiveness. permitirá ao paciente ser responsável da doença. Este tipo de intervenção parece fazer pouco para ajudar o indivíduo a analisar a magnitude e a importância de modificar hábitos alimentares de maneira saudável ao longo prazo.

Conclusão: A intervenção constante por meio da educação nutricional é eficaz na criação de adesão dos pacientes com Diabetes Mellitus tipo 2 ao tratamento nutricional ao longo prazo.

Palavras-chave: Intervenções dietéticas; Diabetes mellitus; adesão, alimentos e educação alimentar e nutricional; avaliação; eficácia.
Universidad Internacional Iberoamericana, Campeche, México.

2 Universidad de Ixtlahuaca CUI, Ixtlahuaca, Estado de México, México.

*Correspondencia: Claudia N. Orozco-González

nelly.orozco@unini.edu.mx

\section{INTRODUCCIÓN}

La modernización de la sociedad contemporánea ha incrementado la prevalencia de obesidad y diabetes mellitus tipo 2 (DM2) en países desarrollados y en vías de desarrollo, como es el caso de México ${ }^{(1)}$. La DM2 es uno de los principales problemas epidemiológicos mundiales debido a la naturaleza compleja de la enfermedad, resultado de sus características multidimensionales y multifactoriales. Más allá de las repercusiones en el incremento de los costos en salud pública, se incluyen en su evolución implicaciones médicas, sociales y psicológicas ${ }^{(2)}$.

Los tratamientos para la DM2 son diversos. Sin embargo, la mejoría e innovación de los mismos sigue siendo necesaria. La alimentación y la actividad física en el marco de estilos de vida saludables se han implementado en pacientes con DM2 como parte de su tratamiento y han reflejado cambios positivos en los marcadores bioquímicos de los individuos, además de una disminución en la presión sanguínea y el peso corporal. No obstante, el ejercicio debe ser prescrito adecuadamente y de acuerdo con las características de cada paciente con la finalidad de reducir riesgos tales como eventos cardiovasculares o daño a los tejidos suaves y articulaciones, entre otros ${ }^{(3)}$.

\footnotetext{
3 Centro de Investigación y Tecnología Industrial de Cantabria, España.

4 Universidad Autónoma de Guadalajara, Guadalajara, Jalisco, México.

5 Nutrir México, México.
}

A pesar de los esfuerzos para mejorar y diversificar los tratamientos, la eficacia de los mismos se ve mermada frecuentemente por la nula o escasa adherencia de los pacientes a los mismos. Se ha observado que las causas de una pobre o nula adherencia a tratamientos nutricionales o farmacológicos gira en torno a tres ejes principales: la enfermedad por sí misma, la actitud del paciente y la actitud del profesional de la salud ${ }^{(3)}$. El objetivo de esta revisión es identificar, a través del análisis de la literatura, la eficacia de las diferentes intervenciones nutricionales en pacientes con DM2 en la creación de adherencia al tratamiento dietético.

\section{MÉTODOS}

Para la búsqueda de literatura se consultaron las bases de datos Pubmed, Scielo, Google Académico, Ovid, Web of Science, Science Direct y Scopus. Se identificaron artículos científicos que analizaran la adherencia a tratamientos nutricionales en pacientes con DM2. Las siguientes palabras y frases clave en inglés fueron utilizadas durante la búsqueda: "Diabetes type", "nutrition", "treatment", "adherence", “menu”, "meal plan", “diet" y "strict diet". En español se incluyeron las palabras y frases: "diabetes mellitus tipo 2", "adherencia nutricional", "menú de alimentación", "dieta estricta" y "nutrición". 
La búsqueda inicial en Google Académico se realizó en español con la palabra "diabetes" y más tarde fue añadida la frase "diabetes mellitus tipo 2". Después, se incluyó el operador "AND" y "adherencia al tratamiento". La oración "diabetes mellitus tipo 2 y adherencia al tratamiento nutricional” se utilizó en ambos idiomas para limitar los resultados. Durante la revisión, aquellos artículos que indicaran adherencia a tratamientos farmacéuticos o psicológicos fueron eliminados. A continuación, se buscó la frase "diabetes mellitus tipo 2 y adherencia a planes de alimentación”, misma que arrojó resultados significativos. Fueron también eliminados aquellos trabajos que se trataran de artículos de revisión, carteles de investigación o estudios tipo metaanálisis. La siguiente base de datos seleccionada fue Pubmed, en la que se encontraron resultados más relevantes. La primera búsqueda fue la frase en inglés "diabetes mellitus type 2 and restricted menu". Se realizó otra búsqueda con el grupo de palabras: "diabetes mellitus type 2 and hypoglycemic menu", de la cual solo se seleccionó un artículo. Con la finalidad de reducir los resultados a trabajos más significativos, la última combinación de palabras buscadas en Pubmed fue "diabetes mellitus y plan de alimentación".

Una vez que se examinaron los artículos se inició la sistematización de los datos. Se elaboraron tres matrices de acuerdo con diferentes objetivos y se capturaron los datos para analizarlos a profundidad. La primera matriz abarca aquellos artículos que mostraron adherencia dietética a través del uso de educación en nutrición; la segunda, se realizó para analizar la adherencia dietética a través de menús o planes de alimentación establecidos y la tercera, fue elaborada para describir la adherencia al tratamiento nutricional a través de educación en nutrición combinada con prescripción de menús y planes de alimentación estrictos o restrictivos. Estas matrices incluyen la metodología empleada, la población objetivo y resultados importantes de los estudios. El 41,4 \% de los artículos revisados abordaron las intervenciones a través de educación en nutrición; el 48,3\%, las intervenciones con menús estrictos o restrictivos; y el 10,3 \% emplearon una combinación de ambos tipos de intervención.

Se encontraron algunos artículos en español e inglés que no fueron incluidos en el estudio, pues los autores no fueron específicos acerca del tipo de análisis, la metodología utilizada o los resultados no eran claros sobre si la intervención llevada a cabo fue exclusivamente psicológica o farmacológica.

\section{RESULTADOS}

\section{Intervención a través de educación en nutrición}

En este grupo se incluyeron 12 artículos en los cuales se exploran intervenciones con educación en nutrición. En ellos se emplearon diferentes estrategias para asegurar la adherencia (Tabla 1).

Por una parte, se observó que la edad de la población elegida influenció la adherencia a la intervención. Por ejemplo, las personas mayores son conscientes y conocen su condición y complicaciones, así como el tratamiento dietético para hacerle frente. Sin embargo, aún con este conocimiento, la adherencia a las indicaciones de su plan de alimentación no fue adecuada, pues asocian cualquier tipo de dieta con restricciones o la sensación de hambre durante el día ${ }^{(4)}$. Por otra parte, este mismo tipo de intervención se implementó con éxito en pacientes del sexo femenino debido a que son ellas quienes, en su mayor parte, están a cargo de la nutrición en el hogar. Mientras que priorizan altamente la calidad de la dieta, sus dificultades se expresan en la selección de porciones de los alimentos y su principal barrera es la condición económica ${ }^{(5)}$.

Muñoz y colaboradores ${ }^{(6)}$ utilizaron herramientas didácticas e instrumentos como videos en diferentes sesiones. Además, utilizaron el optimismo para mostrar una perspectiva diferente acerca de la enfermedad y promover cambios positivos en los estilos de vida. Lo anterior resultó en un porcentaje de adherencia al tratamiento nutricional del $48 \%$. De acuerdo con Pérez y colaboradores ${ }^{(7)}$, la adaptación del método de enseñanza, como resultado de la participación activa del paciente, permite el aprendizaje sin importar la edad, escolaridad o cronicidad de la enfermedad.

Para Arceaga y Celada ${ }^{(8)}$, quienes usaron 2 diferentes estrategias para lograr la adherencia al tratamiento nutricional, las características sociodemográficas y culturales no son determinantes importantes al momento de mantener la adherencia a la intervención. Sin embargo, el tipo de educación empleada para lograr cambios benéficos en el paciente es de la más alta importancia. La educación participativa resultó ser el método ideal para incrementar el conocimiento sobre la DM2 y la adherencia al tratamiento, lo cual, en consecuencia, mejoró los niveles de glucosa del paciente. El proceso también creó conciencia en el paciente, lo que provocó la sensación de control sobre la enfermedad logrando un cambio voluntario. 
Tabla 1. Matriz de educación nutricional

\begin{tabular}{|c|c|c|c|}
\hline Referencia & Diseño de estudio & Población & Hallazgos importantes \\
\hline $\begin{array}{l}\text { Troncoso y } \\
\text { colaboradores, } \\
2008^{(4)}\end{array}$ & $\begin{array}{l}\text { Estudio cualitativo } \\
\text { fenomenológico }\end{array}$ & $\begin{array}{l}\text { Adultos } \\
\text { mayores } \\
(65 \text { años })\end{array}$ & $\begin{array}{l}\text { Los pacientes poseen conocimientos acerca de la DM2, incluidas sus } \\
\text { complicaciones y tratamiento. El conocimiento no significa adherencia. }\end{array}$ \\
\hline $\begin{array}{l}\text { Zanetti y } \\
\text { colaboradores, } \\
2015^{(5)}\end{array}$ & $\begin{array}{l}\text { Estudio } \\
\text { observacional } \\
\text { transversal }\end{array}$ & 421 pacientes & $\begin{array}{l}\text { Los pacientes no siguieron las recomendaciones nutricionales. Este } \\
\text { estudio mostró una asociación entre el sexo (femenino) y la adherencia a } \\
\text { la dieta. }\end{array}$ \\
\hline $\begin{array}{l}\text { Muñoz y } \\
\text { colaboradores, } \\
2012^{(6)}\end{array}$ & Estudio piloto & $\begin{array}{l}27 \text { pacientes } \\
\text { (35-70 años) }\end{array}$ & $\begin{array}{l}\text { Tasa de adherencia: } 48 \% \text {, educación nutricional constante e incremento } \\
\text { en los conocimientos acerca de la DM2 a través de videos. }\end{array}$ \\
\hline $\begin{array}{l}\text { Pérez y } \\
\text { colaboradores, } \\
2009^{(7)}\end{array}$ & $\begin{array}{l}\text { Estudio } \\
\text { cuasiexperimental }\end{array}$ & 90 pacientes & $\begin{array}{l}\text { La educación es esencial para lograr adherencia. Se utilizó motivación } \\
\text { para garantizar un metabolismo regular y estilos de vida saludables. No se } \\
\text { debe brindar solo una fase informativa, sino continuar con una educación } \\
\text { constante mientras que el paciente adquiere hábitos a largo plazo. }\end{array}$ \\
\hline $\begin{array}{l}\text { Arcega- } \\
\text { Domínguez y } \\
\text { colaboradores, } \\
2008^{(8)}\end{array}$ & $\begin{array}{l}\text { Estudio } \\
\text { cuasiexperimental }\end{array}$ & 202 pacientes & $\begin{array}{l}\text { Dos tipos de educación: } \\
\text { - Participación educativa, trabajo sobre autoconocimiento y } \\
\text { conocimiento de estilos de vida: se logró aumento en el conocimiento, } \\
\text { adherencia y voluntad. } \\
\text { - Educación tradicional con conferencias, rotafolios y cuestionarios: se } \\
\text { logró el control de niveles glucémicos. }\end{array}$ \\
\hline $\begin{array}{l}\text { Castro-Cornejo } \\
\text { y colaboradores, } \\
2014^{(9)}\end{array}$ & $\begin{array}{l}\text { Ensayo clínico } \\
\text { aleatorizado }\end{array}$ & $\begin{array}{l}370 \text { pacientes } \\
\text { ambulatorios }\end{array}$ & $\begin{array}{l}\text { Hubo una tasa de adherencia del } 50 \% \text { en el grupo experimental. El grupo } \\
\text { de control no siguió las recomendaciones. Para lograr la adherencia fue } \\
\text { necesario un seguimiento constante por parte del profesional de la salud. }\end{array}$ \\
\hline $\begin{array}{l}\text { Calderón y } \\
\text { colaboradores, } \\
2003^{(10)}\end{array}$ & Ensayo clínico & $\begin{array}{l}107 \text { pacientes, } \\
\text { la mayoría de } \\
\text { ellos mujeres } \\
\text { mayores de } \\
50 \text { años }\end{array}$ & $\begin{array}{l}\text { Aumento de niveles de conocimiento, habilidades para el autocuidado y } \\
\text { la alimentación. No se logró adherencia a largo plazo y la } \mathrm{HbA}_{1 c} \text { volvió a } \\
\text { los valores iniciales. }\end{array}$ \\
\hline $\begin{array}{l}\text { González y } \\
\text { colaboradores, } \\
2015^{(11)}\end{array}$ & $\begin{array}{l}\text { Estudio } \\
\text { cuasiexperimental }\end{array}$ & 56 pacientes & $\begin{array}{l}\text { Adherencia reducida debido a malos hábitos arraigados en la población } \\
\text { en relación con la ingesta alimentaria. El conocimiento sobre diabetes y } \\
\text { dieta correcta aumentó hasta en un } 100 \% \text {. }\end{array}$ \\
\hline $\begin{array}{l}\text { Flores-López y } \\
\text { colaboradores, } \\
2008^{(12)}\end{array}$ & $\begin{array}{l}\text { Estudio } \\
\text { cuasiexperimental }\end{array}$ & $\begin{array}{l}16 \text { pacientes } \\
\text { (32-78 años) }\end{array}$ & $\begin{array}{l}\text { Buena adherencia. Influencia positiva de los talleres de educación } \\
\text { participativos y seguimiento constante. Mejoría del conocimiento, } \\
\text { autocontrol y responsabilidad de los pacientes sobre la enfermedad. }\end{array}$ \\
\hline $\begin{array}{l}\text { Fernández y } \\
\text { colaboradores, } \\
2012^{(13)}\end{array}$ & $\begin{array}{l}\text { Estudio } \\
\text { cuasiexperimental }\end{array}$ & $\begin{array}{l}76 \text { pacientes; } \\
3 \text { hombres y } \\
45 \text { mujeres } \\
(30-70 \text { años })\end{array}$ & $\begin{array}{l}\text { Se consigue un mejor control glucémico. Hubo mayor conocimiento, } \\
\text { autocontrol y manejo del estrés. Buena adherencia gracias a sesiones } \\
\text { constantes de educación. }\end{array}$ \\
\hline $\begin{array}{l}\text { Padilla y } \\
\text { colaboradores, } \\
1997^{(14)}\end{array}$ & $\begin{array}{l}\text { Estudio } \\
\text { cuasiexperimental }\end{array}$ & 19 pacientes & Adherencia gradual. Cambios dietéticos y metabólicos positivos. \\
\hline $\begin{array}{l}\text { Ariza y } \\
\text { colaboradores, } \\
2011^{(15)}\end{array}$ & $\begin{array}{l}\text { Ensayo clínico } \\
\text { aleatorizado }\end{array}$ & $\begin{array}{l}120 \text { pacientes, } \\
64 \text { mujeres y } \\
44 \text { hombres }\end{array}$ & $\begin{array}{l}\text { Se utilizó motivación, educación sobre el ejercicio y sobre nutrición en } \\
\text { grupos e individualmente. } \\
\text { Motivación + actividad física + educación grupal = más adherencia. }\end{array}$ \\
\hline
\end{tabular}

Castro y colaboradores ${ }^{(9)}$ concluyeron que la educación es esencial para prevenir complicaciones, pero que debe ser reforzada con estrategias versátiles. Su método probó, con tasas de hasta un $50 \%$, que la adherencia a la intervención nutricional es posible. Sin embargo, en su estudio la adherencia se obtiene a través de la constante supervisión e instrucción de profesionales de la salud y no de manera voluntaria por el paciente. 
Calderón y colaboradores ${ }^{(10)}$ y González y colaboradores $^{(11)}$ encontraron, en diferentes estudios, que la información sobre DM2 es determinante para lograr el control y la adherencia al tratamiento. La información debe obtenerse a través de intervenciones educativas de largo plazo preferiblemente. En la misma investigación, Calderón y colaboradores ${ }^{(10)}$ concluyeron que los beneficios del control y adherencia terminan al mismo tiempo que la intervención. Ambos están de acuerdo con que los hábitos dietéticos de cada paciente están arraigados en su estilo de vida y pueden ser difíciles de modificar a corto plazo.

Los talleres de educación participativa fueron parte de los estudios cuasiexperimentales de Flores y colaboradores $^{(12)}$, Fernández y colaboradores ${ }^{(13)}$, y Padilla y colaboradores ${ }^{(14)}$. Todos concordaron en que las sesiones de educación nutricional no deben durar más de 60 minutos. En el caso de Fernández y colaboradores ${ }^{(12)}$, y Padilla y colaboradores ${ }^{(14)}$, la intervención duró 6 meses y ambos obtuvieron resultados benéficos respecto del control y la adherencia al tratamiento nutricional, así como un aumento en el conocimiento de la enfermedad, autocontrol, control del estrés, buena calidad de vida y apoyo social. Padilla y colaboradores ${ }^{(14)}$ consideraron que la adherencia fue significativa en el control de consumo de hidratos de carbono con un porcentaje del $63 \%$. Esta adherencia fue gradual y generó un cambio positivo en la percepción del paciente respecto de su propia enfermedad. Flores y colaboradores ${ }^{(12)}$ reportaron una intervención de 10 sesiones, una sesión por día. Esto trajo resultados en el corto plazo, se disminuyeron los niveles de hemoglobina glucosilada $\left(\mathrm{HbA}_{1 \mathrm{c}}\right)$ y se generó conciencia en el paciente respecto de la DM2. Todo lo anterior benefició la adherencia nutricional de manera significativa. Padilla y colaboradores ${ }^{(14)}$ y Flores y colaboradores ${ }^{(12)}$ afirmaron que la adherencia nutricional está basada en la responsabilidad conjunta entre el paciente y el equipo de profesionales de la salud. Esto transforma el proceso en un flujo de información continua, clara y simple que es transmitida al paciente en una interacción constante.

Finalmente, Ariza y colaboradores ${ }^{(15)}$ emplearon herramientas tales como la motivación, controles estrictos, ejercicio y educación dietética a nivel grupal e individual para poder obtener una adecuada adherencia y, de este modo, se obtuvo un buen control con los pacientes en grupos. E incluso, cuando los pacientes también recibieron prescripciones de actividad física, la adherencia se duplicó, lo cual resultó también en un control metabólico incrementado.

\section{Intervención por menú estricto}

En este grupo se incluyeron 14 artículos en los cuales se les entregó a los pacientes de cada uno de los grupos de investigación un menú preestablecido (Tabla 2).

Las investigaciones de Martínez y colaboradores ${ }^{(16)}$ y Garay-Sevilla y colaboradores ${ }^{(17)}$ comparten el mismo diseño de estudio, aunque los resultados publicados por cada uno de ellos fueron sumamente diferentes. Los resultados de Martínez y colaboradores ${ }^{(16)}$ hicieron poco énfasis en la adherencia dietética mostrada por sus pacientes con dietas generales y estrictas. No obstante, su porcentaje de adherencia fue del $62 \%$ y ayudó en el control glucémico. Por su parte, GaraySevilla y colaboradores ${ }^{(17)}$ enfatizaron bastante en la adherencia dietética; pero a pesar de ello, en su estudio la adherencia fue baja. De acuerdo con los autores, esto sucedió porque los profesionales sanitarios tuvieron que enfrentar las ideas y creencias preconcebidas de los pacientes respecto de la medicina convencional y tradicional. El estudio mostró que una concepción fatalista, la negación de la situación y el nivel socioeconómico son determinantes en la adherencia. El apoyo familiar también tiene una influencia en los resultados y se propuso que hay un mayor apoyo al proveedor económico de la familia, lo que deja en segundo lugar al paciente.

En su investigación, Rentería y colaboradores ${ }^{(18)}$ consideraron factores como el estado emocional y los pensamientos que pudieran influenciar el consumo de alimentos, horarios y contextos de convivencia $y$ ambiente al construir y determinar un plan de alimentación que genere una verdadera adherencia. Sin embargo, en su estudio la adherencia fue medida a través del índice de masa corporal (IMC), lo cual disminuye la veracidad sobre la adherencia dietética. En su estudio también se hace referencia a intervenciones psicopedagógicas, pero no se deja claro cómo se evaluó el componente psicológico.

Leiva y colaboradores ${ }^{(19)}$ fragmentaron la dieta en 5 tiempos de comida, obteniendo una adherencia variable en cada uno de ellos. Los porcentajes más altos de adherencia se presentaron en el consumo del desayuno y el almuerzo, con un $100 \%$; la cena mostró tasas de adherencia del $45 \%$ y las colaciones matutinas y vespertinas reportaron $72 \%$ y $62 \%$, respectivamente. Aun cuando la adherencia fue reportada, esta no mostró ninguna asociación positiva a un buen control metabólico ni a la disminución de los niveles de $\mathrm{HbA}_{1 c}$.

Calpe-Cristino y colaboradores ${ }^{(20)}$ realizaron un ensayo clínico aleatorizado en el que se muestra que la 
Tabla 2. Matriz de plan de alimentación

\begin{tabular}{|c|c|c|c|}
\hline Año/autor & $\begin{array}{l}\text { Diseño de } \\
\text { estudio }\end{array}$ & Población & Hallazgos importantes \\
\hline $\begin{array}{l}\text { Martínez- } \\
\text { Domínguez y } \\
\text { colaboradores, } \\
2011^{(16)}\end{array}$ & Estudio transversal & 70 pacientes & $\begin{array}{l}\text { Porcentaje de adherencia a la dieta: } 62,2 \% \text {, poca preocupación por la } \\
\text { dieta, aunque hubo buen control glucémico. }\end{array}$ \\
\hline $\begin{array}{l}\text { Garay-Sevilla y } \\
\text { colaboradores, } \\
2011^{(17)}\end{array}$ & Estudio transversal & $\begin{array}{l}210 \text { pacientes, } \\
159 \text { mujeres y } \\
51 \text { hombres }\end{array}$ & $\begin{array}{l}\text { La adherencia a una dieta específica fue baja y se asoció con la } \\
\text { creencia en la medicina tradicional, con el estilo de vida del paciente y } \\
\text { su nivel de educación. }\end{array}$ \\
\hline $\begin{array}{l}\text { Rentería y } \\
\text { colaboradores, } \\
2017^{(18)}\end{array}$ & $\begin{array}{l}\text { Ensayo clínico } \\
\text { antes-después }\end{array}$ & $\begin{array}{l}100 \text { pacientes, } \\
17 \text { hombres y } \\
83 \text { mujeres }\end{array}$ & $\begin{array}{l}\text { La adherencia al plan de alimentación medido a través del peso y la } \\
\text { masa corporal resultó ser ineficaz. Se lograron actitudes positivas, } \\
\text { motivación constante, autocontrol y resolución de problemas. }\end{array}$ \\
\hline $\begin{array}{l}\text { Leiva y } \\
\text { colaboradores, } \\
2016^{(19)}\end{array}$ & Estudio transversal & $\begin{array}{l}60 \text { sujetos } \\
(30-65 \text { años) }\end{array}$ & $\begin{array}{l}\text { Adherencia: } \\
\text { - } 100 \% \text { para desayuno y almuerzo } \\
\text { - } 72 \% \text { para colación matutina } \\
\text { - } 62 \% \text { para colación vespertina } \\
\text { - } 45 \% \text { para cena } \\
\text { No hubo asociación positiva con el control metabólico. Cuanto más } \\
\text { fragmentada fue la dieta, hubo mayor aporte calórico. }\end{array}$ \\
\hline $\begin{array}{l}\text { Calpe-Cristino y } \\
\text { colaboradores, } \\
2017^{(20)}\end{array}$ & $\begin{array}{l}\text { Ensayo clínico } \\
\text { aleatorizado }\end{array}$ & 19 pacientes & $\begin{array}{l}\text { Se logró adherencia a la dieta y empatía. Los menús individualizados } \\
\text { de } 1500 \text { y } 1400 \text { calorías, y gráficos aumentaron las tasas de } \\
\text { adherencia. }\end{array}$ \\
\hline $\begin{array}{l}\text { Bader y } \\
\text { colaboradores, } \\
2013^{(21)}\end{array}$ & $\begin{array}{l}\text { Piloto de } \\
\text { intervención }\end{array}$ & 33 pacientes & $\begin{array}{l}\text { Porcentaje de adherencia dietética: } 54 \% \text {. Se empleó un programa } \\
\text { de internet sin control que los pacientes podían modificar. Veracidad } \\
\text { dudosa de los resultados. }\end{array}$ \\
\hline $\begin{array}{l}\text { Cunningham y } \\
\text { colaboradores, } \\
2006^{(22)}\end{array}$ & $\begin{array}{l}\text { Ensayo clínico } \\
\text { aleatorizado }\end{array}$ & $\begin{array}{l}10 \text { pacientes } \\
(42-61 \text { años })\end{array}$ & $\begin{array}{l}\text { Adherencia del } 20 \% \text { y } 2 \text { semanas más de intervención voluntaria. Se } \\
\text { lograron conocimientos sobre porciones, autocontrol y alimentos con } \\
\text { bajo índice glucémico. }\end{array}$ \\
\hline $\begin{array}{l}\text { Veleba y } \\
\text { colaboradores, } \\
2016^{(23)}\end{array}$ & $\begin{array}{l}\text { Estudio } \\
\text { cuasiexperimental }\end{array}$ & $\begin{array}{l}77 \text { pacientes, } \\
47 \% \text { hombres y } \\
53 \% \text { mujeres }\end{array}$ & $\begin{array}{l}\text { Adherencia del } 55 \% \text {. Menú vegetariano y actividad física constante. } \\
\text { Durante el estudio se entregaron alimentos, porciones y horario de } \\
\text { comidas. }\end{array}$ \\
\hline $\begin{array}{l}\text { Hussain y } \\
\text { colaboradores, } \\
2012^{(24)}\end{array}$ & $\begin{array}{l}\text { Estudio } \\
\text { observacional } \\
\text { exploratorio }\end{array}$ & $\begin{array}{l}363 \text { pacientes, } \\
86 \text { hombres y } \\
277 \text { mujeres. } 102 \\
\text { pacientes con DM2 }\end{array}$ & $\begin{array}{l}\text { Dieta cetogénica controlada durante } 24 \text { semanas. Hubo control } \\
\text { metabólico. }\end{array}$ \\
\hline $\begin{array}{l}\text { Ponzo y } \\
\text { colaboradores, } \\
2017^{(25)}\end{array}$ & $\begin{array}{l}\text { Estudio } \\
\text { observacional } \\
\text { exploratorio }\end{array}$ & 500 pacientes & $\begin{array}{l}\text { La adherencia al plan de alimentación se logró en un tercio de } \\
\text { los pacientes. La actitud y la individualización de la dieta estricta } \\
\text { determinaron la adherencia. }\end{array}$ \\
\hline $\begin{array}{l}\text { Imai y } \\
\text { colaboradores, } \\
2011^{(26)}\end{array}$ & $\begin{array}{l}\text { Ensayo clínico } \\
\text { aleatorizado }\end{array}$ & 101 pacientes & $\begin{array}{l}\text { Adherencia inestable (duración del estudio: } 24 \text { meses). El consumo de } \\
\text { verduras antes del consumo de hidratos de carbono simples resultó } \\
\text { significativo. Se consiguió el control glucémico. }\end{array}$ \\
\hline $\begin{array}{l}\text { Balas-Nakas y } \\
\text { colaboradores, } \\
2010^{(27)}\end{array}$ & Ensayo clínico & $\begin{array}{l}69 \text { mujeres } \\
(22-42 \text { años })\end{array}$ & $\begin{array}{l}\text { Adherencia del } 41,9 \% \text { en mujeres con diabetes gestacional. } \\
\text { Insistencia en mantener el control glucémico. En mujeres con DM2; } \\
\text { mala actitud, bajo interés y baja adherencia. }\end{array}$ \\
\hline $\begin{array}{l}\text { Soria-Contreras } \\
\text { y colaboradores, } \\
2014^{(28)}\end{array}$ & Estudio piloto & $\begin{array}{l}15 \text { pacientes } \\
\text { (35-80 años) }\end{array}$ & $\begin{array}{l}\text { Adherencia de } 5 \text { días a la semana a un menú estricto de } 2000 \mathrm{kcal} \text {. } \\
\text { Asesoramiento individualizado a cada paciente y viabilidad para el } \\
\text { control de los niveles glucémicos. }\end{array}$ \\
\hline
\end{tabular}


cultura y el contexto de cada paciente son cruciales en el tratamiento y la prescripción dietética. Se utilizaron menús gráficos con alimentos especiales y porciones para mantener un estricto control. Los menús se mezclaron con dietas de $1500 \mathrm{kcal}$ y 21 porciones de hidratos de carbono y $1800 \mathrm{kcal}$ y 24 porciones de hidratos de carbono. El estudio respetó la preparación típica de comida pakistaní y la adaptó al plan de alimentación para una persona con DM2. Este estudio también consideró la religión del grupo, de esta manera se reportó haberse logrado adherencia a la dieta y una empatía de los pacientes hacia las indicaciones nutricionales.

En su estudio piloto, Bader y colaboradores ${ }^{(21)}$ lograron una adherencia a la dieta en un $54 \%$ al permitir a cada paciente la automonitorización a través del uso de un programa de internet. Pese a ello, los resultados sobre el control glucémico y los hábitos dietéticos son dudosos ya que, aunque se establecieron planes de alimentación, no se realizó un control sobre los pacientes y su consumo de alimentos, lo que vuelve a los resultados imprecisos.

Cunningham y colaboradores ${ }^{(22)}$ lograron que los pacientes incluidos en su ensayo clínico aleatorizado continuaran con su tratamiento dietético, con una adherencia voluntaria al plan por más de dos semanas. Se encontró que, aunque solamente el $20 \%$ de ellos logró adherencia a la dieta, todos adquirieron conocimientos acerca de porciones, autocontrol y alimentos de bajo índice glucémico.

Veleba y colaboradores ${ }^{(23)}$ y Hussain y colaboradores $^{(24)}$ en diferentes estudios propios utilizaron menús estrictos y control continuo. Por una parte, Veleba y colaboradores ${ }^{(23)}$ utilizaron un plan de alimentación vegetariano y actividad física constante con el que se logró un $55 \%$ de adherencia a la dieta; los alimentos fueron proporcionados al paciente en las porciones correctas y en los tiempos adecuados, lo que facilitó una mayor adherencia. Por otra parte, Hussain y colaboradores $^{(24)}$ emplearon una dieta cetogénica controlada que disminuyó positivamente los niveles de $\mathrm{HbA}_{1 c^{\prime}}$ glucosa, colesterol, triglicéridos en la sangre y peso corporal en un lapso de 24 semanas. Se logró, además, una adherencia estadísticamente significativa.

Una actitud positiva, disposición y claridad en el menú fueron determinantes para alcanzar una adherencia correcta en el estudio exploratorio observacional de Ponzo y colaboradores ${ }^{(25)}$, en el que casi la tercera parte de la población estudiada se adhirió al plan de alimentación. Los pacientes prefirieron un plan de alimentación definido y no recomendaciones generales.
Imai y colaboradores ${ }^{(26)}$ mostraron en una prueba controlada aleatorizada que consumir verduras antes de los hidratos de carbono simples en su menú tenía un impacto significativo en el control de los niveles glucémicos, pero la adherencia a la dieta se afectó con el paso del tiempo. Después de 24 meses, algunos pacientes abandonaron el menú o no se adhirieron a él correctamente.

Durante su ensayo clínico, Balas-Nakash y colaboradores $^{(27)}$ obtuvieron una adherencia significativa de un $41,9 \%$ en mujeres con diabetes gestacional, en comparación con mujeres con DM2. Así mismo, el estudio mostró que las mujeres con diabetes gestacional se esforzaron por mantener el control glucémico con mayor insistencia y tenían una correcta percepción de la adherencia a su dieta, en comparación con las mujeres que presentaban DM2.

Finalmente, Soria y colaboradores ${ }^{(28)}$ lograron una adherencia significativa de al menos 5 días a la semana a un plan de alimentación de $2000 \mathrm{kcal}$ por 4 semanas. También se incrementó la disposición a la adherencia a través de asesoramiento individual.

\section{Intervención con menú estricto y educación nutricional}

Rodríguez y colaboradores ${ }^{(29)}$, Lancheros y colaboradores ${ }^{(30)}$, y Morrison y colaboradores ${ }^{(31)}$ comparten la idea de que para lograr una correcta adherencia a la dieta se debe combinar la educación nutricional con la prescripción de un menú establecido. Sin embargo, Rodríguez y colaboradores ${ }^{(29)}$ fueron los únicos que lograron una correcta adherencia al utilizar una combinación de estas intervenciones. Lancheros y colaboradores ${ }^{(30)}$ y Morrison y colaboradores ${ }^{(31)}$ obtuvieron resultados variados en sus estudios transversales. Lancheros y colaboradores ${ }^{(30)}$ reportaron que cuanto más restrictivo es el plan, mayor es la sobrecompensación del consumo de ciertos macronutrientes. Mientras tanto, Morrison y colaboradores $^{(31)}$, quienes tuvieron a mujeres embarazadas en su estudio, experimentaron mala adherencia y actitudes negativas hacia el plan de alimentación y la educación brindadas. El estudio discute esto como resultado de la falta de preocupación de las mujeres embarazadas acerca del riesgo de desarrollar diabetes gestacional (Tabla 3).

\section{DISCUSIÓN}

El primer paso en el tratamiento de la DM2 es establecer una alimentación que no solo satisfaga las necesidades de cada paciente, sino que también logre cambios 
Tabla 3. Matriz de educación nutricional y menú estricto

\begin{tabular}{|l|l|l|l|}
\hline \multicolumn{1}{|c|}{ Año/autor } & Diseño de estudio & \multicolumn{1}{c|}{ Población } & \multicolumn{1}{c|}{ Hallazgos importantes } \\
\hline $\begin{array}{l}\text { Rodríguez y } \\
\text { colaboradores, 2013(29) }\end{array}$ & $\begin{array}{l}\text { Ensayo clínico } \\
\text { antes-después }\end{array}$ & $\begin{array}{l}90 \text { pacientes } \\
\text { (34-86 años) }\end{array}$ & $\begin{array}{l}\text { Adherencia positiva combinando estrategias. } \\
\text { Cambio de actitud en la preferencia del paciente por un } \\
\text { menú estricto. }\end{array}$ \\
\hline $\begin{array}{l}\text { Lancheros y } \\
\text { colaboradores, 2010(30) }\end{array}$ & $\begin{array}{l}\text { Estudio transversal } \\
\text { descriptivo }\end{array}$ & $\begin{array}{l}30 \text { personas, } \\
60 \% \text { mujeres y } \\
40 \% \text { hombres }\end{array}$ & $\begin{array}{l}\text { Baja adherencia a las recomendaciones calóricas y de } \\
\text { macronutrientes. Cuanto más restrictivo es el plan, mayor } \\
\text { sobrecompensación del sujeto en la adecuación de la } \\
\text { ingesta de nutrientes. }\end{array}$ \\
\hline $\begin{array}{l}\text { Morrison y } \\
\text { colaboradores, 2012 }\end{array}$ & Estudio transversal & $\begin{array}{l}1447 \text { pacientes } \\
\text { embarazadas }\end{array}$ & $\begin{array}{l}\text { La adherencia y la calidad de la dieta fueron bajas a pesar } \\
\text { del riesgo de desarrollar diabetes. Actitud pobre y bajo } \\
\text { interés de algunas pacientes. }\end{array}$ \\
\hline
\end{tabular}

graduales en los hábitos de alimentación del mismo. Los aspectos socioculturales, económicos y familiares influyen de manera importante en el proceso de cambio y, por consiguiente, deben ser fundamentales en la conformación de un tratamiento personalizado para los pacientes. De acuerdo con Khazrai y colaboradores ${ }^{(32)}$, existen muchos planes de alimentación disponibles para que los pacientes con DM2 puedan elegir uno basado en sus gustos personales y tradiciones culturales. Es importante proveer una dieta personalizada cuando sea posible con el fin de incrementar la eficacia de la misma en la reducción de los síntomas de la diabetes y fomentar la adherencia del paciente.

La efectividad en el tratamiento nutricional implica corresponsabilidad entre el paciente y el personal sanitario. Por una parte, el nutriólogo o nutricionista debe ser la principal fuente de información sobre una dieta adecuada para el paciente $y$, por otra parte, el paciente debe hacerse cargo de su propia salud. Adicionalmente, en una revisión de los factores psicosociales en la adherencia al tratamiento y el autocontrol de la DM2 se indicó que "la falta de adherencia a las recomendaciones puede ser involuntaria o intencional, reflejando el proceso de toma de decisiones por parte del paciente, quien valora los beneficios y los beneficios anticipados relacionados con el tratamiento y costos del mismo y su calidad de vida"(33).

Troncoso y colaboradores ${ }^{(34)}$ identificaron una escasez de profesionales en el área médica, específicamente médicos y nutricionistas, y la rotación del personal de salud como situaciones que alteran la continuidad y compromiso con la adherencia terapéutica. Esta idea también es apoyada por Ortiz y colaboradores ${ }^{(35)}$ al relacionar las variables de atención médica, apoyo social y adherencia al tratamiento ${ }^{(35,36)}$. Entre los problemas sobre los profesionales de la salud se encuentran que, además de estar poco entrenados para convencer a los pacientes de la importancia de la adherencia al plan alimentario, se tienen pocas referencias sobre cómo hacer una medición más eficiente sobre esta variable.

De las intervenciones dietéticas analizadas, la que resultó más efectiva fue la educación participativa, ya que esta técnica involucra al paciente como un agente para el desarrollo de sus propios conocimientos y promueve que el individuo interiorice lo aprendido de manera teórica para luego aplicarlo en la rutina diaria. Esta práctica fomenta la toma de decisiones saludables en relación con el autocuidado y, de este modo, mejora la calidad de vida. La intervención debe ser constante y de largo plazo. Una vez que el paciente es consciente de su enfermedad, tomará responsabilidad sobre el control de la misma. La educación a largo plazo provee conocimientos que el paciente puede utilizar como forma de empoderamiento para la toma de decisiones; sin embargo, estos estudios demostraron cómo la libertad de elegir los alimentos disminuye de manera considerable la adherencia nutricional ${ }^{(4-6,8-12)}$.

La intervención a través de menús establecidos o un control estricto genera buenos resultados a corto plazo, pero apenas logran la consciencia que le permitirá al paciente ser responsable de la enfermedad. Este tipo de intervención parece hacer poco para ayudar al individuo a analizar la magnitud e importancia de modificar los hábitos dietéticos de forma saludable a largo plazo $^{(16-22)}$.

Se encontró poca información sobre intervenciones de educación nutricional combinadas con la prescripción de un menú, lo que causó que los autores de esta 
revisión cuestionaran la efectividad de dicha combinación para lograr una correcta adherencia nutricional. Es importante revisar opciones de evaluación correcta e individualizadas para el paciente, ya que una correcta inspección del contexto del sujeto (situación económica, tiempo de preparación de alimentos, educación nutricional, gustos, entre otros) podría ayudar a comprender la estrategia ideal para el paciente.

Un dato interesante es que solo unos pocos estudios reportaron el factor psicológico como un punto importante para la adherencia al tratamiento. Por ejemplo, Balas-Nakash y colaboradores ${ }^{(27)}$ propusieron que los diferentes diseños de investigación para la evaluación de la adherencia deben tomar en cuenta el factor psicológico, pero su estudio no reporta la evaluación de este factor. Asimismo, aunque Pérez y colaboradores ${ }^{(7)}$ consideraron que para tener una mejor adherencia al tratamiento es importante estar en las mejores condiciones psicológicas posibles, su estudio tampoco evalúa este componente. Finalmente, Lancheros y colaboradores $^{(30)}$ reportaron la evaluación de la actitud con el apoyo de un psicólogo, pero no reportó ningún tratamiento psicológico que mejore la adherencia.

También es importante recalcar que entre las limitaciones de esta revisión se encontró que la comparación de resultados está limitada por las diferencias metodológicas en la selección de participantes y por el tipo de instrumento utilizado para recolectar datos sobre el patrón dietético ${ }^{(37,38)}$. Sin embargo, la población incluida en los estudios revisados tenía características sociodemográficas comunes con estudios similares realizados en América Latina, donde la población con DM2 se conforma por personas de 60 años de edad, predominantemente femenina, con sobrepeso y con un nivel educativo primario, lo que relaciona directamente su adherencia al tratamiento nutricional ${ }^{(39,40)}$.

La adherencia es un proceso largo y constante, y es un reto lograr una adecuada intervención nutricional; por esto, este artículo es una invitación a seguir diseñando estrategias educativas y de control que realmente beneficien al paciente con DM2.

\section{CONCLUSIONES}

Esta revisión se centró en comparar dos estrategias para mejorar la adherencia al tratamiento nutricional: el componente educativo y la restricción dietética. La educación a largo plazo provee conocimientos que el paciente puede utilizar como forma de empoderamiento para la toma de decisiones, pero los estudios demostraron cómo la libertad de elegir los alimentos disminuye de manera considerable la adherencia nutricional. La intervención a través de menús establecidos o un control estricto genera buenos resultados a corto plazo, pero no suscitan en el paciente la concientización del problema de modo que le permita ser responsable de la enfermedad. Es decir, no le permite al individuo reconocer la importancia de modificar los hábitos dietéticos de forma saludable a largo plazo para el control de la DM2.

\section{Declaración de autoría}

Orozco y Vieyra contribuyeron igualmente a la concepción y diseño de la investigación, adquisición y análisis de los datos, e interpretación de los datos. Todos los autores redactaron y revisaron el manuscrito. Acuerdan ser plenamente responsables de garantizar la integridad y precisión del trabajo, y leyeron y aprobaron el manuscrito final.

\section{Conflicto de interés}

Los autores declaran no tener intereses en competencia por el contenido de este artículo.

\section{Recursos financieros}

El presente estudio no fue financiado.

\section{Referencias bibliográficas}

1. Trejo-Gutiérrez JF. Epidemiología del síndrome metabólico y diabetes mellitus tipo 2: ¿El diluvio que viene? Arch Cardiol Mex. 2004;74(Supl 2):S267-70.

2. Gonzáles-Castro P. El apoyo familiar en la adherencia al tratamiento nutricional del paciente con diabetes mellitus tipo 2 (DM2). Waxapa. 2011;2(5):102-6.

3. Figuerola D. Implicación del paciente con diabetes tipo 2 en el autocuidado de su enfermedad: un reto pendiente. Av en Diabetol. 2009;25(6):501-2.

4. Troncoso PC, Sotomayor CM, Ruiz HF, Zúñiga TC. Interpretación de los conocimientos que influyen en la adherencia a la dietoterapia en adultos mayores con Diabetes Tipo 2 de una comuna rural. Rev Chil Nutr. 2008;35(4):421-26. doi: 10.4067/S0717-75182008000500004

5. Zanetti ML, Arrelias ACC, Franco RC, Dos Santos MA, Rodrigues LFF, Faria GHT. Adherence to nutritional recommendations and sociodemographic variables in patients with diabetes mellitus. Rev Esc Enferm USP. 2015;49(4):619-25. doi: 10.1590/S0080-623420150000400012.

6. Muñoz MSG, Lerman GI, Cuevas D, Aguilar SC, Velasco ML, Hernández JS, et al. Estudio piloto del beneficio de la utiliza- 
ción de un video como herramienta educativa en pacientes con diabetes tipo 2. Rev Endocrinol Nutr. 2012;20(2):56-62.

7. Pérez DA, Alonso CL, García MAJ, Garrote RI, González PS, Morales RJM. Intervención educativa en diabéticos tipo 2. Rev Cubana de Med Gen Integr. 2009;25(4):17-29.

8. Arceaga-Domínguez A, Celada-Ramírez NA. Control de pacientes con diabetes. Impacto de la educación participativa versus educación tradicional. Rev Med Inst Mex Seguro Soc. 2008;46(6):685-90.

9. Castro-Cornejo MA, Rico-Herrera L, Padilla-Raygoza N. Efecto del apoyo educativo para la adherencia al tratamiento en pacientes con diabetes tipo 2: un estudio experimental. Enferm Clin. 2014;24(3):162-167. Doi: 10.1016/j. enfcli.2013.11.004

10. Calderón TJR, Solís VJ, Castillo SOA, Cornejo AMP, Figueroa DV, Paredes J, et al. Efecto de la educación en el control metabólico de pacientes con diabetes mellitus tipo 2 del Hospital Nacional Arzobispo Loayza. Rev Soc Peru Med Interna. 2003;16(1):17-25.

11. González RR, Cardentey GJ, Casanova MMC. Intervención sobre educación nutricional en pacientes con diabetes mellitus tipo 2. Rev Arch Med Camagüey. 2015;19(3):262-69.

12. Flores-López ME, Velázquez-Tlapanco J, Camacho-Calderón N. Control metabólico, estado nutricional y presión arterial de diabéticos tipo 2. Eficacia de una estrategia educativa. Rev Med Inst Mex Seguro Soc. 2008;46(3):301-10.

13. Fernández VA, Abdala CTA, Alvara SEP, Tenorio FGL, López VE, Cruz CS, et al. Estrategias de autocuidado en pacientes con diabetes mellitus tipo 2. Rev Esp Méd Quir. 2012;17(2):94-99.

14. Padilla G, Aráuz AG, Roselló M. Metodología para evaluar la adherencia a la dieta en diabetes mellitus no insulino dependiente [Internet]. Rev Costarric Cienc Med.1997;18(4):15-28.

15. Ariza CC, Gavara PV, Muñoz UA, Aguera MF, Soto MM, Lorca SJR. Mejora en el control de los diabéticos tipo 2 tras una intervención conjunta: educación diabetológica y ejercicio físico. Aten Primaria. 2011;43(8):398-406. Doi: 10.1016/j.aprim.2010.07.006

16. Martínez-Domínguez GI, Martínez-Sánchez LM, RodríguezGázquez MA, Agudelo-Vélez CA, Jiménez-Jiménez JG, VargasGrisales N, et al. Adherencia terapéutica y control metabólico en pacientes con Diabetes Mellitus Tipo 2, pertenecientes a una institución hospitalaria, de la ciudad de Medellín (Colombia), año 2011. Arch Med. 2014;14(1):44-50.

17. Garay-Sevilla ME, Porras JS, Malacara JM. Coping strategies and adherence to treatment in patients with type 2 diabetes mellitus. Rev Invest Clin. 2011;63(2):155-61.

18. Rentería RA, Rodríguez CML, García RJC. Programa de adherencia a la dieta en sujetos con diabetes mellitus tipo 2 y su efecto en los índices de peso y masa corporal: estudio exploratorio. Rev Elec Psic Iztacala. 2017;20(2):479-501.

19. Leiva T, Basfi-fer K, Rojas P, Carrasco F, Ruz OM. Efecto del fraccionamiento de la dieta y cantidad de hidratos de carbono en el control metabólico en pacientes con diabetes mellitus tipo 2, sin terapia con insulina. Rev Med Chile. 2016;144(10):1247-53. doi: 10.4067/S003498872016001000002 .

20. Calpe-Crisitino A, Fructuoso-González E, OchandoAymerich M, Fernández-Martín I, Rodero-Nuño M, HidalgoOrtiz M, et al. Habilidades comunicativas de enfermería para conseguir dietas adaptadas efectivas en pacientes paquistaníes con Diabetes mellitus 2. Rev Esp Comun Salud. 2017;8(1):519. Doi: https://doi.org/10.20318/recs.2017.3602

21. Bader A, Gougeon R, Joseph L, Da Costa D, Dasgupta K. Nutritional Education Through Internet-Delivered Menu Plans Among Adults with Type 2 Diabetes Mellitus: Pilot Study. JMIR Res Protoc. 2013;2(2):e41. doi: 10.2196/resprot. 2525

22. Cunningham C, Johnson S, Cowell B, Soroudi N, SegalIsaacson CJ, Davis NJ, et al. Menu Plans in a Diabetes Self-management weight loss program. J Nutr Educ Behav. 2006;38(4):264-6. doi: 10.1016/j.jneb.2006.01.013

23. Veleba J, Matoulek M, Hill M, Pelikanova T, Kahleova H. "A Vegetarian vs. Conventional Hypocaloric Diet: The Effect on Physical Fitness in Response to Aerobic Exercise in Patients with Type 2 Diabetes." A Parallel Randomized Study. Nutrients. 2016;8(11):671. Doi: 10.3390/nu8110671

24. Hussain TA, Mathew TC, DashtiAA, Asfar S, Al-Zaid N, Dashti HM. Effect of low-calorie versus low-carbohydrate ketogenic diet in type 2 diabetes. Nutrition. 2012;28(10):1016-21. doi: 10.1016/j.nut.2012.01.016

25. Ponzo V, Rosato R, Tarsia E, Goitre I, De Michieli F, Fadda M, et al. Self-reported adherence to diet and preferences towards type of meal plan in patient with type 2 diabetes mellitus. A cross-sectional study. Nutr Metab Cardiovasc Dis. 2017; 27(7): 642-50. Doi: 10.1016/j.numecd.2017.05.007.

26. Imai S, Matsuda M, Hasegawa G, Fukui M, Obayashi H, Ozasa $\mathrm{N}$, et al. A simple meal plan of 'eating vegetables before carbohydrate' was more effective for achieving glycemic control than an exchange-based meal plan in Japanese patients with type 2 diabetes. Asia Pac J Clin Nutr. 2011;20(2):161-8.

27. Balas-Nakash M, Rodríguez-Cano A, Muñoz-Manrique C, Vásquez-Peña P, Perichart-Perera O. Tres métodos para medir la adherencia a un programa de terapia médica y nutricia en mujeres embarazadas con diabetes y su asociación con el control glucémico. Rev Inv Clin. 2010;62(3):235-43.

28. Soria-Contreras DC, Bell RC, McCargar LJ, Chan CB. Feasibility and Efficacy of Menu Planning combined with individual counselling to improve health outcomes and dietary adherence in people with Type 2 Diabetes: A pilot study. Can J Diabetes. 2014;38(5):320-5. doi: 10.1016/j. jcjd.2014.03.009

29. Rodríguez CML, Rentería RA, García RJC. Adherencia a la dieta en pacientes diabéticos: efectos de una intervención. Summa Psic UST. 2013;10(1):91-101

30. Lancheros PL, Pava CA, Bohórquez PA. Identificación de la adherencia al tratamiento nutricional aplicando el modelo de 
Conocimientos, Actitudes y Prácticas en un grupo de personas con Diabetes Mellitus tipo 2 atendidas en la Asociación Colombiana de Diabetes. Diaeta. 2010;28(133):17-23

31. Morrison MK, Koh D, Lowe JM, Miller YD, Marshall AL, Colyvas K, et al. Postpartum diet quality in Australian women following a gestational diabetes pregnancy. Eur J Clin Nutr. 2012;66(10):1160-5. doi: 10.1038/ejcn.2012.84

32. Khazrai YM, Defeudis G, Pozzilli P. Effect of diet on type 2 diabetes mellitus: a review. Diabetes Metab Res Rev. 2014;30(Suppl 1):24-33. doi: 10.1002/dmrr.2515

33. Gonzalez JS, Tanenbaum ML, Commissariat PV. Psychosocial factors in medication adherence and diabetes self-management: Implications for research and practice. Am Psychol. 2016;71(7):539-51. Doi: 10.1037/a0040388

34. Troncoso PC, Delgado SD, Rubilar VC. Adherencia al tratamiento en pacientes con Diabetes Mellitus tipo 2. Rev Costarr Salud Pública. 2013;22:9-13.

35. Ortiz M, Ortiz E, Gatica A, Gómez D. Factores Psicosociales Asociados a la Adherencia al Tratamiento de la Diabetes Mellitus tipo 2. Terapia Psicológica. 2011;29(1):5-11. doi: 10.4067/S0718-48082011000100001

36. Mumu SJ, Saleh F, Ara F, Afnan F, Ali L. Non-adherence to life-style modification and its factors among type 2 diabetic patients. Indian J Public Health. 2014;58(1):40-4. doi: 10.4103/0019-557X.128165
37. Handelsman Y, Bloomgarden ZT, Grunberger G, Umpierrez G, Zimmerman RS, Bailey TS, et al. American Association of Clinical endocrinologists and American College of Endocrinology - clinical practice guidelines for developing a diabetes mellitus comprehensive care plan - 2015-Executive summary. Endocr Pract. 2015;21(Suppl 1):1-87. doi: 10.4158/EP15672.GL

38. Muñoz-Pareja M, León-Muñoz LM, Guallar-Castillón P, Graciani A, López-García E, Banegas JR, et al. The Diet of Diabetic Patients in Spain in 2008-2010: Accordance with the Main Dietary Recommendations-A Cross-Sectional Study. PLoS One. 2012;7(6):e39454. doi: 10.1371/journal. pone.0039454

39. Hermoza ARD, Matellini MBM, Rosales RAJ, Noriega RVH. Adherencia a terapia médica nutricional en pacientes con diabetes mellitus tipo 2, de un hospital nacional de nivel III en Lima, Perú. Rev Med Hered. 2017;28(1):150-6. doi: https:// doi.org/10.20453/rmh.v28i3.3181

40. Ganiyu AB, Mabuza LH, Malete NH, Govender I, Ogunbanjo GA. Non-adherence to diet and exercise recommendations amongst patients with type 2 diabetes mellitus attending Extension II Clinic in Botswana. Afr J Prim Health Care Fam Med. 2013;5(1):457. doi: 10.4102/phcfm.v5i1.457. 